\title{
Analyzing the Causes of Blood Donor Deferrals and Characteristics of Deffered Individuals in Kurdistan Province, Iran
}

\author{
Hosein Hatami ${ }^{1,2}$, Mahtab Maghsoodlu², Pejman Salehifar ${ }^{2,3}$, Mohammad Saied Karimian $^{2,3^{*}}$, Shirin Ferdowsi ${ }^{*}$ \\ 'Department of Public Health, School of Health, Shahid Beheshti University of Medical Sciences, Tehran, Iran \\ ${ }^{2}$ Blood Transfusion Research Center, High Institute for Research and Education in Transfusion Medicine, Tehran, Iran \\ ${ }^{3}$ Kurdistan Blood Transfusion Center, Sanandaj, Iran
}

\author{
*Correspondence to \\ Shirin Ferdowsi, \\ Email: ferdowsishirin@gmail.com; \\ Mohammad Saied Karimian, \\ Tel: +982188601501 \\ Fax: +982188601555 \\ Email: ms_karimian@irimc.org
}

Received April 13, 2018

Accepted June 1, 2018

Published online September 30, 2018

\begin{abstract}
Introduction: Donor recruitment is an essential step in blood banking. Accordingly, this study aimed to evaluate donor deferral rate, causes of deferrals, and characteristics of deferred individuals in Kurdistan province located in west of Iran.

Methods: This was a cross-sectional study carried out in Kurdistan Blood Transfusion Center from 2010 to 2015. A total of 175845 donors were selected to be screened. They were physically examined and detailed interviews were conducted with all the donors. In addition, standard operating procedures (SOPs) were used for donor selection and deferral.

Results: According to the obtained results, the deferral rate was $14.55 \%$ ( 25586 out of 175845 ). The top 7 deferral reasons included the use of certain medications $(n=3996,15.6 \%)$, abnormal blood pressure $(n=2311,9.03 \%)$, polycythemia $(n=2177,8.5 \%)$, risky behaviors $(n=1848$, $7.2 \%)$, previous donation in the last 3 months $(n=1512,5.9 \%)$, history of repeatedly reactive tests for infectious agents $(n=1334,5.21 \%)$, and anemia $(n=1243,4.85 \%)$. Meanwhile, the overall rate of deferral was higher in donors $<35$ years old $(P<0.001)$, females $(P<0.001)$, firsttime donors $(P<0.001)$, unmarried $(P<0.001)$, and less than 12 years of schooling $(P<0.001)$. Conclusion: The findings of this study indicated that the main reason for deferral was related to medications. Therefore, public education regarding health requirements of blood donors can be effective in reducing the number of deferrals.

Keywords: Blood donation, Deferral reasons, Blood donors
\end{abstract}

Please cite this article as follows: Hatami $\mathrm{H}$, Maghsoodlu M, Salehifar P, Karimian MS, Ferdowsi S. Analyzing the causes of blood donor deferrals and characteristics of deffered individuals in Kurdistan province Iran. Int J Basic Sci Med. 2018;3(3):114119. doi:10.15171/ ijbms.2018.21.

\section{Introduction}

Donor screening process is a crucial step to increase donaed blood saftey. ${ }^{1}$ The purpose of blood donor screening is to minimize the risk of infections transmitted by blood products. ${ }^{2}$ Both temporary and permanent bood donor deferrals result in a shrinkage in donor pools and low blood components available for transfusion. ${ }^{3}$ Consequently, there should be an awareness regarding the causes of blood donation deferrals. Moreover, identification of the reasons for deferrals can lead to modifications in blood safety programs and recruitment strategies. $^{4}$

Blood donor deferral prevalence has been reported from $5.6 \%$ to $35.6 \%$ in different countries. ${ }^{3-11}$ This difference in prevalence pertentage of deferral may be attributed to various donor selection criteria due to differences in donor population and geographic distribution of transfusiontransmitted infections (TTIs). ${ }^{12}$ The donor deferral rate in several cities of Iran has been reported as $25.6 \%$ (Isfahan), ${ }^{8} 26.5 \%$ (Ilam), ${ }^{13} 22.5 \%$ (Tehran), ${ }^{14}$ and $30.9 \%$ (Shiraz). ${ }^{15}$ According to what has been mentioned, the aim of this study was to determine the prevalence and reasons for blood donor deferrals in Kurdistan Province. To the best of the researchers' knowledge, this study is the first report analyzing the rate and reason of donor deferral in Kurdistan.

\section{Materials and Methods \\ Donor Screening Strategy}

This cross-sectional epidemiologic

(C) 2018 The Author(s); Published by Zabol University of Medical Sciences. This is an open-access article distributed under the terms of the Creative Commons Attribution License (http://creativecommons.org/licenses/by/4.0), which permits unrestricted use, distribution, and reproduction in any medium, provided the original work is properly cited. 
study was performed in Kurdistan Blood Transfusion Organization during 2010-2015. All the donors underwent a screening process to determine their eligibility for donation. The standard operating procedures (SOPs) developed by the safe blood transfusion programme (SBTP) were applied for donor screening. ${ }^{16}$ The screening process included identification, medical interview as well as blood pressure and hemoglobin measurements. Post-donation serologic analysis contained tests to detect hepatitis B virus (HBV), hepatitis C virus (HCV), human immunodeficiency virus (HIV), and syphilis. Regular donors were considered based on their previous history of blood donation while first-time donors were defined as those without any records of blood donation. Besides, individuals who did not meet the donor selection criteria were deferred temporarily or permanently. Deferral rates, frequencies of different medical reasons for deferral, and demographic details were collected. Subjcts who failed to donate, due to inability in venous access or vasovagal reaction, were not considered as defferals since these donors were otherwise eligible.

\section{Statistical Analysis}

The SPSS software, version 16 was run to analyse the data. In addition, the chi-square test was used to assess a significant relationship between the categorical variables.
The significance level was set at 5\% $(P<0.05)$.

Results

A total of 175845 blood donors were presented during the study period comprising of 55339 (31.4\%) first-time and $120506(68.5 \%)$ regular donors. Of the total number of donors, 160433 (91.2\%) of them were males while 15412 (8.76\%) were females. Deferrals occurred in 25586 (14.55\%) blood donors. The highest (15.6\%) and lowest (11.9\%) deferral rates belonged to the years 2012 and 2015, respectively. That is, 5153 (2012) and 3950 (2015) individuals were deferred, respectively. The results are provided in Table 1.

The deferral rate had a decreasing trend from 2012 to 2015. Among the individuals, 23294 (91\%) were deferred temporarily while 2292 ( $8.95 \%)$ of them were deferred permanently. The overall proportion of deferrals was higher among those less than 35 years old $(P<0.001)$, females $\quad(P<0.001)$, first-time donors $(P<0.001)$, unmarried $(P<0.001)$, and less than 12 years of schooling $(P<0.001)$ (Table 2). Overall, 19829 men $(77.4 \%)$ and 5757 women $(22.5 \%)$ were deferred. In other words, men were deferred more than women. However, women had a higher deferral rate (5657 out of $15412,37.35 \%)$ compared to men (19829 out of $160433,12.35 \%)$. Totally, the top 7 deferral reasons were as follows: the use of

Table 1. Blood Deferral Rate During 2010-2015 in Kurdistan Blood Transfusion Organization

\begin{tabular}{|c|c|c|c|c|}
\hline Year & Blood Donors (N) & $\begin{array}{c}\text { Number of Temporary Deferrals } \\
\text { No. (\%) }\end{array}$ & $\begin{array}{l}\text { Number of Permanent Deferrals } \\
\text { No. }(\%)\end{array}$ & $\begin{array}{c}\text { Total } \\
\text { No. }(\%)\end{array}$ \\
\hline 2010 & 29539 & $3766(12.74)$ & $252(0.85)$ & $4018(13.6)$ \\
\hline 2011 & 30388 & $4125(13.57)$ & $498(1.63)$ & $4623(15.21)$ \\
\hline 2012 & 30507 & $4766(15.62)$ & $387(1.26)$ & $5153(16.89)$ \\
\hline 2013 & 26445 & 3412 (12.9) & $349(1.31)$ & $3761(14.22)$ \\
\hline 2014 & 29125 & $3661(12.56)$ & $420(1.44)$ & $4081(14.01)$ \\
\hline 2015 & 29841 & $3564(11.94)$ & $386(1.29)$ & $3950(13.23)$ \\
\hline Total & 845175 & $23294(13.24)$ & $2292(1.30)$ & $25586(14.55)$ \\
\hline
\end{tabular}

Table 2. Demographic Features of Deffered Donors in Kurdistan Blood Transfusion Organization

\begin{tabular}{|c|c|c|c|c|c|}
\hline \multicolumn{2}{|c|}{ Demographic Characteristics } & \multirow{3}{*}{$\begin{array}{c}\text { Number of Temporary Deferrals (\%) } \\
10854(19.6) \\
12440(10.32)\end{array}$} & \multirow{3}{*}{$\begin{array}{l}\boldsymbol{P} \text { Value } \\
<0.001\end{array}$} & \multirow{3}{*}{$\begin{array}{c}\text { Number of Permanent Deferrals (\%) } \\
816(1.47) \\
1476(1.22)\end{array}$} & \multirow{3}{*}{$\begin{array}{l}\boldsymbol{P} \text { Value } \\
<0.001\end{array}$} \\
\hline Donation & First-time & & & & \\
\hline status & Regular & & & & \\
\hline \multirow{2}{*}{ Gender } & Female & $5405(35.07)$ & \multirow{2}{*}{$<0.001$} & $352(2.28)$ & \multirow{2}{*}{$<0.001$} \\
\hline & Male & $17889(11.15)$ & & $1940(1.2)$ & \\
\hline \multirow{5}{*}{ Age (y) } & $18-25$ & $2133(9.1)$ & \multirow{5}{*}{$<0.001$} & $132(5.75)$ & \multirow{5}{*}{$<0.001$} \\
\hline & $25-35$ & $8123(34.8)$ & & $485(21.1)$ & \\
\hline & $35-45$ & $5775(24.7)$ & & $460(20.06)$ & \\
\hline & $45-55$ & $4661(20.0)$ & & $598(26.09)$ & \\
\hline & $55-65$ & $2602(11.1)$ & & $617(26.91)$ & \\
\hline \multirow{2}{*}{ Education } & High school or lower & $16388(22.9)$ & \multirow{2}{*}{$<0.001$} & $1584(2.2)$ & \multirow{2}{*}{$<0.001$} \\
\hline & Academic & $6906(6.15)$ & & $708(0.6)$ & \\
\hline \multirow{2}{*}{$\begin{array}{l}\text { Marital } \\
\text { status }\end{array}$} & Married & $16734(11.68)$ & \multirow{2}{*}{$<0.001$} & $1719(1.2)$ & \multirow{2}{*}{$<0.001$} \\
\hline & Single & $6560(16.24)$ & & $573(1.4)$ & \\
\hline
\end{tabular}


certain medications (3996, 15.6\%), abnormal blood pressure $(2311,9.03 \%)$, polycythemia $(2177,8.5 \%)$, risky behaviors $(1848,6.3 \%)$, previous donation in the last 3 months $(1512,5.9 \%)$, history of repeatedly reactive tests for infectious agents (1334, 5.21\%), and anemia (1243, $4.85 \%)$.
The main reasons for deferrals among male donors were being on medication (3377, 17.03\%), abnormal blood pressure (1735, 8.7\%), and risky behaviors (1588, $8.08 \%$ ) while in female donors, polycythemia (1417, $24.6 \%)$, anemia (962,16.7\%), and being on medication $(619,10.7 \%)$ caused the highest deferral reasons (Table 3).

Table 3. Causes of Blood Donor Deferrals in Kurdistan Blood Transfusion Organization

\begin{tabular}{|c|c|c|c|c|c|c|c|c|c|c|c|c|c|c|}
\hline \multirow{2}{*}{$\begin{array}{l}\text { Blood Donation Deferral } \\
\text { Reasons }\end{array}$} & \multicolumn{2}{|c|}{2010} & \multicolumn{2}{|c|}{2011} & \multicolumn{2}{|c|}{2012} & \multicolumn{2}{|c|}{2013} & \multicolumn{2}{|c|}{2014} & \multicolumn{2}{|c|}{2015} & \multicolumn{2}{|c|}{ Total } \\
\hline & $\mathbf{M}$ & $\mathbf{F}$ & $\mathbf{M}$ & $\mathbf{F}$ & $\mathbf{M}$ & $\mathbf{F}$ & $\mathbf{M}$ & $\mathbf{F}$ & $\mathbf{M}$ & $\mathbf{F}$ & $\mathbf{M}$ & $\mathbf{F}$ & $\mathbf{M}$ & $\mathbf{F}$ \\
\hline Medications & 456 & 91 & 808 & 100 & 96 & 149 & 693 & 66 & 652 & 113 & 672 & 100 & 3377 (17.03) & $619(10.7)$ \\
\hline Abnormal blood pressure & 178 & 69 & 247 & 101 & 346 & 143 & 564 & 123 & 285 & 83 & 115 & 57 & $1735(8.7)$ & $576(10.0)$ \\
\hline Anemia & 80 & 189 & 56 & 168 & 60 & 133 & 26 & 61 & 23 & 31 & 36 & 191 & $281(1.4)$ & $962(16.7)$ \\
\hline Polycythemia & 168 & 49 & 37 & 197 & 76 & 337 & 76 & 302 & 223 & 481 & 180 & 51 & $760(3.8)$ & $1417(24.6)$ \\
\hline Bloodletting & 96 & 20 & 184 & 16 & 198 & 31 & 123 & 14 & 101 & 7 & 153 & 2 & $855(4.3)$ & $126(2.1)$ \\
\hline Risky behaviors & 98 & 3 & 496 & 7 & 444 & 5 & 204 & 2 & 162 & 4 & 184 & 5 & $1588(8.08)$ & $26(0.45)$ \\
\hline Cardiovascular diseases & 69 & 35 & 126 & 18 & 134 & 25 & 150 & 20 & 96 & 11 & 42 & 21 & $617(3.1)$ & $13(2.2)$ \\
\hline Surgery & 31 & 10 & 43 & 9 & 67 & 9 & 57 & 6 & 43 & 10 & 41 & 3 & $282(1.4)$ & $47(0.8)$ \\
\hline $\begin{array}{l}\text { Tattooing, acupuncture, or } \\
\text { ear piercing }\end{array}$ & 10 & 2 & 16 & 5 & 12 & 5 & 16 & 7 & 13 & 5 & 153 & 30 & $220(1.1)$ & $64(1.1)$ \\
\hline Drug abuse & 18 & 4 & 79 & 1 & 113 & 1 & 56 & 0 & 61 & 0 & 62 & 0 & 389 (1.96) & $6(0.1)$ \\
\hline Weight & 3 & 6 & 3 & 9 & 10 & 9 & 4 & 8 & 7 & 13 & 8 & 12 & $35(0.17)$ & $57(0.99)$ \\
\hline Allergies & 4 & 3 & 14 & 6 & 13 & 7 & 3 & 2 & 7 & 12 & 0 & 0 & $41(0.2)$ & $30(0.52)$ \\
\hline Elderly & 60 & 3 & 24 & 6 & 23 & 8 & 32 & 4 & 103 & 10 & 62 & 0 & 304 (1.5) & $31(0.53)$ \\
\hline Kidney disease & 1 & 2 & 9 & 0 & 3 & 0 & 11 & 0 & 6 & 1 & 143 & 96 & $173(0.87)$ & $99(1.7)$ \\
\hline Skin problem & 25 & 1 & 42 & 2 & 53 & 4 & 33 & 3 & 38 & 10 & 1 & 1 & $192(0.96)$ & $21(0.36)$ \\
\hline Respiratory diseases & 128 & 25 & 203 & 18 & 145 & 25 & 142 & 23 & 134 & 16 & 2 & 1 & $754(3.8)$ & $108(1.87)$ \\
\hline Travel & 1 & 2 & 5 & 0 & 6 & 0 & 1 & 0 & 3 & 0 & 3 & 0 & $19(0.09)$ & $2(0.03)$ \\
\hline $\begin{array}{l}\text { History of repeatedly } \\
\text { reactive test }\end{array}$ & 101 & 13 & 181 & 29 & 110 & 23 & 75 & 8 & 103 & 12 & 657 & 22 & $1227(6.1)$ & $107(1.85)$ \\
\hline Dental procedure & 19 & 3 & 56 & 3 & 45 & 6 & 35 & 2 & 7 & 3 & 46 & 2 & $208(1.04)$ & $19(0.33)$ \\
\hline Vaccination & 19 & 4 & 14 & 0 & 10 & 1 & 7 & 1 & 2 & 0 & 9 & 2 & $61(0.3)$ & $8(0.13)$ \\
\hline $\begin{array}{l}\text { Contact with hepatitis } \\
\text { patient }\end{array}$ & 7 & 0 & 5 & 8 & 2 & 12 & 1 & 2 & 0 & 0 & 3 & 1 & $32(0.16)$ & $23(0.39)$ \\
\hline Renal diseases & 10 & 2 & 8 & 0 & 3 & 0 & 11 & 0 & 6 & 1 & 111 & 96 & $149(0.75)$ & $99(1.7)$ \\
\hline Infectious diseases & 16 & 5 & 39 & 13 & 39 & 17 & 26 & 9 & 66 & 8 & 162 & 14 & $340(1.7)$ & $66(1.14)$ \\
\hline Nervous system disorders & 41 & 10 & 53 & 14 & 92 & 13 & 94 & 10 & 63 & 10 & 25 & 4 & $360(1.8)$ & $61(1.05)$ \\
\hline $\begin{array}{l}\text { Malignancy/ } \\
\text { chemotherapy }\end{array}$ & 0 & 0 & 3 & 0 & 2 & 0 & 0 & 0 & 5 & 0 & 0 & 0 & $10(0.05)$ & $0(0)$ \\
\hline Occupational limitation & 2 & 2 & 6 & 1 & 14 & 0 & 6 & 0 & 5 & 1 & 5 & 0 & $38(0.19)$ & $4(0.06)$ \\
\hline Thrombosis & 1 & 2 & 2 & 1 & 6 & 3 & 5 & 2 & 4 & 2 & 0 & 0 & $18(0.09)$ & $10(0.17)$ \\
\hline $\begin{array}{l}\text { Blood donation interval of } \\
3 \text { months }\end{array}$ & 157 & 30 & 277 & 32 & 394 & 21 & 247 & 19 & 235 & 16 & 63 & 21 & $1373(6.9)$ & $139(2.4)$ \\
\hline Prison history & 14 & 2 & 20 & 1 & 19 & 3 & 10 & 1 & 7 & 0 & 9 & 0 & $79(0.3)$ & $7(0.12)$ \\
\hline Diabetes \& on insulin & 2 & 5 & 21 & 8 & 16 & 7 & 13 & 0 & 25 & 3 & 0 & 0 & $77(0.3)$ & $23(0.39)$ \\
\hline Blood disorders & 4 & 2 & 6 & 2 & 6 & 2 & 5 & 0 & 9 & 2 & 11 & 5 & $41(0.2)$ & $13(0.22)$ \\
\hline Thyroid disorder & 12 & 10 & 36 & 20 & 33 & 31 & 41 & 18 & 32 & 16 & 0 & 0 & $154(0.77)$ & $95(1.65)$ \\
\hline Fasting & 20 & 7 & 5 & 0 & 24 & 4 & 33 & 7 & 40 & 9 & 11 & 2 & $133(0.67)$ & $29(0.5)$ \\
\hline Endoscopy & 37 & 10 & 43 & 12 & 28 & 5 & 45 & 6 & 37 & 2 & 57 & 13 & $247(1.24)$ & $48(0.83)$ \\
\hline Fever & 0 & 2 & 2 & 1 & 5 & 2 & 3 & 1 & 2 & 1 & 4 & 1 & $16(0.08)$ & $8(0.13)$ \\
\hline Others & 940 & 567 & 459 & 187 & 919 & 556 & 163 & 23 & 449 & 161 & 125 & 42 & 3055 (15.4) & $1536(26.6)$ \\
\hline Total & 40 & & 46 & & & & 37 & & & & & & 2558 & \\
\hline
\end{tabular}


Table 4. Deferral Rates and Their Frequencies in Different Studies

\begin{tabular}{|c|c|c|}
\hline Studies & Deferral Rate & Common Deferral Reasons \\
\hline $\begin{array}{l}\text { Iran, Kurdistan (present } \\
\text { study) }\end{array}$ & $14.55 \%$ & $\begin{array}{l}\text { Medications }(15.6 \%) \text {, abnormal blood pressure }(9.03 \%) \text {, polycythemia }(8.5 \%) \text {, risky behaviors }(6.3 \%) \text {, } \\
\text { previous donation in last } 3 \text { months }(5.9 \%) \text {, history of repeatedly reactive test }(5.21 \%) \text {, anemia }(4.85 \%)\end{array}$ \\
\hline $\begin{array}{l}\text { Kasraian etal, Shiraz, } \\
\text { Iran }^{15}\end{array}$ & $30.90 \%$ & High risk behavior $(43.60 \%)$, diseases $(31.90 \%)$, others $(13.50 \%)$ \\
\hline $\begin{array}{l}\text { Birjandi et al, Isfahan, } \\
\text { Iran }^{8}\end{array}$ & $25.6 \%$ & $\begin{array}{l}\text { Abnormal blood pressure }(36.5 \%) \text {, high Hb level }(24 \%) \text {, medication }(23.3 \%) \text {, low Hb level }(21.4 \%) \text {, } \\
\text { travel }(19.8 \%) \text {, bloodletting }(19.7 \%) \text {, high-risk behavior }(19.1 \%)\end{array}$ \\
\hline Maleki et al, Ilam, Iran ${ }^{13}$ & $26.5 \%$ & $\begin{array}{l}\text { Medications }(23.7 \%) \text {, high blood pressure }(21.8 \%) \text {, hematological disorders }(15.5 \%) \text {, bloodletting } \\
(11.9 \%) \text {, common cold }(7.3 \%) \text {, risky behavior }(4.9 \%)\end{array}$ \\
\hline Atar et al, Tehran, Iran ${ }^{14}$ & $22.5 \%$ & Risk of blood born disease (43.36\%), disease/exposure $(34.02 \%)$, medications $(13.91 \%)$ \\
\hline Gajjar et al, India ${ }^{17}$ & $11.16 \%$ & Anemia $(48.33 \%)$, hypertension $(11.94 \%)$, low body weight $(7.95 \%)$, medications $(6.13 \%)$ \\
\hline Arslan et al, Turkey ${ }^{18}$ & $14.6 \%$ & Anemia (20.07\%), common cold $(17.70 \%)$, high-risk behavior $(16.70 \%)$ \\
\hline $\begin{array}{l}\text { Abdelaal et al, Saudi } \\
\text { Arabia }^{19}\end{array}$ & $8.7 \%$ & Persistent high pulse rate $(19 \%)$, poor veins $(17 \%)$, low blood pressure $(12 \%)$ \\
\hline Ekwere et al, Nigeria ${ }^{9}$ & $16 \%$ & Low hemoglobin (39\%), high blood pressure $(3.1 \%)$, under weight $(2.4 \%)$ \\
\hline Gonzo et al, Namibia ${ }^{20}$ & $8.6 \%$ & Anemia $(45.0 \%)$, pregnancy \& breast feeding $(13.7 \%)$, medications $(13.6 \%)$ \\
\hline Tufail et al, Pakistan ${ }^{21}$ & $13.58 \%$ & Anemia (4.9\%), HCV confirmed positive (3.1\%), thrombocytopenia ( $1.8 \%)$, HBsAg positive $(0.81 \%)$ \\
\hline Waheed et al, Pakistan ${ }^{22}$ & $4.3 \%$ & Anemia $(41 \%)$, underweight $(22.5 \%)$, low blood pressure $(5.1 \%)$, under/overage $(5.9 \%)$ \\
\hline Ngoma et al, Japan ${ }^{23}$ & $14 \%$ & $\begin{array}{l}\text { Anemia }(50 \%) \text {, interview decisions }(27 \%) \text {, histories of blood transfusion or transplantation }(57 \%) \text {, risky } \\
\text { behavior }(26 \%)\end{array}$ \\
\hline Rabeya et al, Malaysia ${ }^{24}$ & $5.6 \%$ & $\begin{array}{l}\text { Low hemoglobin }(40.7 \%) \text {, Hypertension }(29.4 \%) \text {, Diseases }(15.6 \%) \text {, Low blood pressure }(3.5 \%) \text {, high } \\
\text { hemoglobin }(1.7 \%) \text {, Short duration of interval between donations }(1.7 \%)\end{array}$ \\
\hline $\begin{array}{l}\text { Charles et al, Trinidad } \\
\text { and Tobago }{ }^{25}\end{array}$ & $35.6 \%$ & High-risk sexual contact $(27.6 \%)$, anemia $(22.2 \%)$, hypertension $(17.5 \%)$ \\
\hline Chenna et al, India ${ }^{26}$ & $5.6 \%$ & Low Hemoglobin (48.1\%), hypertension (16.4\%), underweight $(8.9 \%)$ \\
\hline
\end{tabular}

Table 4 represents a comparison of blood donor deferral rates in various studies in Iran and other countries.

\section{Discussion}

In the present study, the deferral rate of Kurd blood donors for a 6-year long period was estimated as $14.55 \%$. These donors were deferred for various reasons in predonation screening. This deferral rate was lower than those of the most previous reports in $\operatorname{Iran}^{8,13-15}$ and other countries. ${ }^{3,9,25}$ However, lower deferral rates have been reported in Islamabad $(4.3 \%)^{22}$ and Karachi $(12.2 \%)$ in Pakistan ${ }^{27}$, Malaysia (5.6\%), ${ }^{24}$ India (11.16\%), ${ }^{17}$ Saudi Arabia (8.7\%), ${ }^{19}$ and Namibia (8.6\%). ${ }^{20}$ The most common cause of deferral at blood center of the present study was the use of medications (15.6\%). This finding is consistent with the result of a recent report by Maleki et $\mathrm{al}^{13}$ in Ilam (west of Iran).

Certain drugs can reduce the function and applicability of blood cells and products or cause adverse effects in recipients. ${ }^{28}$ Blood donors on eligible medications should be deferred for a period consistent with the pharmacokinetics of the drugs. For example, in cases of anti-platelet drugs consumption, donors are deffered for piroxicam and clopidogrel from 2 to 14 days, respectively. Defferals for aspirin and paracetamol usage have been reported in nearly $5 \%$ of blood donations. ${ }^{29}$ Hypersensitivity reactions due to asprin is regarded as a common concern in donors. Moreover, penicillin induced allergy may be encountered in blood recipients due to anti-penicillin antibodies in donated blood units. ${ }^{29}$

The second most common cause of deferral among Kurd donors was abnormal blood pressure. As regards gender, the highest leading cause of blood donation deferral in males was taking medications (17.03\%) while polycythemia (24.6\%) was found to be the leading deferral reason in females. This finding is in contrast to the findings of several other studies that have reported anemia as the most common cause of deferral especially among women. ${ }^{3,9,17,18,20-24}$ In the present study, anemia was the second common cause of deferral in females and the seventh cause overall.

In addition, the overall deferral rate was higher in firsttime compared to regular donors $(21.08 \%$ vs. $11.54 \%$, respectively). Similarly, the results from a study performed in southwest of Iran (Shiraz) ${ }^{15}$ showed a higher deferral rate among first-time donors $(48.1 \%)$ as compared to repeated donors $(13.1 \%)$. In another study in Isfahan (center of Iran), ${ }^{8}$ first-time donors constituted $76.5 \%$ of the total deferrals compared to $17.5 \%$ in repeated donors.

The findings of the present study also showed that $37.35 \%$ of the deferrals belonged to female donors while only $12.35 \%$ of the referrals was found to be related to male donors. Namely, deferral rates were higher in 
female donors as compared to their male counterparts $(P<0.001)$. This finding is in line with the results obtained by other studies. ${ }^{3,8,17,23,30}$ In the current study, temporary deferrals were responsible for $91.05 \%$ of defferals whereas permanent deferrals accounted for the remaining8.95\% (), which is similar to the findings of Khurram et al. ${ }^{27}$

\section{Conclusion}

Generally, findings of this study indicated that the main reason for blood donation deferral was related to medications affecting donated blood quality or exposing the blood recippients at risk. Therefore, public education regarding the importance of blood donation and donors' conditions can be effective in reducing the number of deferrals.

\section{Ethical Approval}

The present project was approved by the Research Ethics Committees of the Iranian Blood Transfusion Organization (IBTO).

\section{Competing Interests}

None.

\section{Acknowledgements}

The authors acknowledge blood transfusion research center, High Institute for Research and Education in Transfusion Medicine of Tehranand Kurdistan Blood Transfusion Center of Sanandaj.

\section{References}

1. Eder A. Evidence-based selection criteria to protect blood donors. J Clin Apher. 2010;25(6):331-337. doi:10.1002/ jca.20257

2. Qureshi ZM, Naseem L. Patterns of interview-based deferrals of blood donors at a tertiary care hospital. Int J Pathol. 2008;6(2):75-78.

3. Goncalez TT, Sabino EC, Schlumpf KS, et al. Analysis of donor deferral at three blood centers in Brazil. Transfusion. 2013;53(3):531-538. doi:10.1111/j.1537-2995.2012.03820.x

4. Agnihotri N. Whole blood donor deferral analysis at a center in Western India. Asian J Transfus Sci. 2010;4(2):116122. doi:10.4103/0973-6247.67035

5. Custer B, Chinn A, Hirschler NV, Busch MP, Murphy EL. The consequences of temporary deferral on future whole blood donation. Transfusion. 2007;47(8):1514-1523. doi:10.1111/j.1537-2995.2007.01292.x

6. Bashawri LA. A review of predonation blood donor deferrals in a university hospital. J Family Community Med. 2005;12(2):79-84.

7. Bahadur S, Jain S, Goel RK, Pahuja S, Jain M. Analysis of blood donor deferral characteristics in Delhi, India. Southeast Asian J Trop Med Public Health. 2009;40(5):10871091.

8. Birjandi F, Gharehbaghian A, Delavari A, Rezaie N, Maghsudlu M. Blood donor deferral pattern in Iran. Arch Iran Med. 2013;16(11):657-660. doi:0131611/aim.009
9. Ekwere TA, Ino-Ekanem M, Motilewa OO, Ibanga IA. Pattern of blood donor deferral in a tertiary hospital, South-south, Nigeria: A three-year study review. Int J Blood Transfus Immunohematol. 2014;4:7-13. doi:10.5348/ijbti2014-14-OA-2

10. Ngoma AM, Goto A, Nollet KE, Sawamura Y, Ohto H, Yasumura S. Blood Donor Deferral among Students in Northern Japan: Challenges Ahead. Transfus Med Hemother. 2014;41(4):251-256. doi:10.1159/000365406

11. Girish CJ, Chandrashekhar TN, Ramesh BK, Kantikar SM. Pre-donation deferral of whole blood donors in district transfusion centre. J Clin Diagn Res. 2012;6(1):47-50.

12. Mascaretti L, James V, Barbara J, et al. Comparative analysis of national regulations concerning blood safety across Europe. Transfus Med. 2004;14(2):105-112. doi:10.1111/ j.1365-3148.2004.0487.x

13. Maleki F, Mohammadi HR, Taghinejad H, Shoohani M, Amraei M. Study of reasons for exemption for deferral of blood donors in Ilam blood transfusion center. J Basic Res Med Sci. 2015;2(3):10-16.

14. Attarchi Z, Ghafouri M, Hajibaygi B, Assari S, Alavian SM. Donor deferral and blood-borne infections in blood donors of Tehran. The Scientific Journal of Iranian Blood Transfusion Organization. 2006;2(7):353-364.

15. Kasraian L, Negarestani N. Rates and reasons for blood donor deferral, Shiraz, Iran. A retrospective study. Sao Paulo Med J. 2015;133(1):36-42. doi:10.1590/1516-31802013-7110002

16. Zaheer HA, Waheed U. Blood safety system reforms in Pakistan. Blood Transfus. 2014;12(4):452-457. doi:10.2450/2014.0253-13

17. Gajjar H, Shah FR, Shah NR, Shah CK. Whole blood donor deferral analysis at General hospital blood bank-A retrospective study. Medicine (Baltimore). 2014;235:6-13.

18. Arslan O. Whole blood donor deferral rate and characteristics of the Turkish population. Transfus Med. 2007;17(5):379-383. doi:10.1111/j.1365-3148.2007.00738.x

19. Abdelaal M, Anwar F. Analysis of blood donor deferral in Jeddah, Saudi Arabia: Characteristics and causes. J Pak Med Assoc. 2016;66(11):1392-1395.

20. Gonzo M, Shuuveni A, Mukesi M, Chipare I, Moyo S. Assessment of donor deferral rates and deferral reasons at the Namibia blood transfusion services. Int J Blood Transfus Immunohematol. 2016;5:37-43. doi:10.5348/ijbti2016-27-OA-7

21. Tufail S, Babar F, Ikram N, Raza M, Abdul-Shakoor H. Blood donors deferral-causes. J Rawal Med Coll. 2013;17(1):119-121.

22. Waheed U, Zaheer HA. Evaluation of deferral pattern among the blood donors in Islamabad, Pakistan. Glob J Transfus Med. 2016;1(2):81-84. doi:10.4103/24558893.189857

23. Ngoma AM, Goto A, Sawamura Y, Nollet KE, Ohto $\mathrm{H}$, Yasumura S. Analysis of blood donor deferral in Japan: characteristics and reasons. Transfus Apher Sci. 2013;49(3):655-660. doi:10.1016/j.transci.2013.06.020

24. Rabeya Y, Rapiaah M, Rosline H, Ahmed SA, Zaidah WA, Roshan TM. Blood pre-donation deferrals--a teaching hospital experience. Southeast Asian J Trop Med Public Health. 2008;39(3):571-574. 
25. Charles KS, Hughes P, Gadd R, Bodkyn CJ, Rodriguez M. Evaluation of blood donor deferral causes in the Trinidad and Tobago National Blood Transfusion Service. Transfus Med. 2010;20(1):11-14. doi:10.1111/j.13653148.2009.00968.x

26. Chenna D, Shastry S, Murugesan M, Baliga PB. Implication of deferral pattern on the donor pool: Study at a Tertiary Care Hospital. J Appl Hematol. 2015;6(3):111-114. doi:10.4103/1658-5127.165649

27. Khurram S, Borhany M, Anwar N, et al. Frequency and reasons of donor deferral prior to blood donation process: a single centre experience. Transfus Med. 2017;27(1):10-
15. doi:10.1111/tme.12368

28. Becker CD, Stichtenoth DO, Wichmann MG, Schaefer C, Szinicz L. Blood Donors on Medication - an Approach to Minimize Drug Burden for Recipients of Blood Products and to Limit Deferral of Donors. Transfus Med Hemother. 2009;36(2):107-113. doi:10.1159/000203355

29. Ferner RE, Dunstan JA, Chaplin S, Baird GM. Drugs in donated blood. Lancet. 1989;2(8654):93-94.

30. Misje AH, Bosnes V, Heier HE. Gender differences in presentation rates, deferrals and return behaviour among Norwegian blood donors. Vox Sang. 2010;98(3 Pt 1):e241248. doi:10.1111/j.1423-0410.2009.01267.x 